\title{
Metacontrast: Internal contours and different dependent variables
}

\author{
LESTER A. LEFTON and LINDA L. HERNANDEZ \\ University of South Carolina, Columbia, South Carolina 29208
}

\begin{abstract}
Extent of metacontrast was assessed using square-wave black and white gratings as targets. The number of internal contours in the target and delay of the mask were systematically manipulated. Three experiments each used a different dependent variable; Experiment 1 used accuracy, Experiment 2 used duration threshold, and Experiment 3 used category estimations. Results showed, (1) targets with many internal contours are difficult to see, (2) there were no backward enhancement effects, (3) targets with few contours and targets with many contours, when detected, are relatively easy to mask, (4) targets with intermediate numbers of contours $(2.54$ to 8 cycles/deg) are relatively easy to see and produce monotonic metacontrast functions, but, (5) category estimation measures showed some evidence of $\mathrm{U}$-shaped metacontrast functions. The results are discussed in terms of task differences.
\end{abstract}

Metacontrast is affected by the nature of the stimuli used, particularly stimuli with internal contours (Dember, Mathews, \& Stefl, 1973). Targets with internal contours in a no-mask detection task are harder to detect. Both duration threshold (Dember et al., 1973) and accuracy measures (Lefton, 1974) have shown that as the number of internal contours in a target increases the targets are more difficult to detect. Moreover, Dember has found backward enhancement effects and also that certain targets are virtually unmaskable (Cox \& Dember, 1970; Dember \& Stefl, 1972; Ellis \& Dember, 1971). Portions of Dember's results have been replicated (Lefton, 1974).

The present investigation was conducted in an attempt to further explore metacontrast using squarewave black and white gratings as targets. In all three studies gratings varied in number of internal contours and the interval between the target and mask was varied. The purpose of the studies was to test the generality of previous findings obtained with square-wave gratings as internal contours (Dember, Stefl, \& Kao, 1974) as well as to further investigate any potential enhancement effects that might be obtained with striped stimuli which were not obtained with the pie-shaped segmented stimuli primarily used in the past.

\section{GENERAL METHOD}

\section{Subjects}

Twelve graduate and two undergraduate students at the University of South Carolina served as subjects. All were paid volunteers and all had normal or corrected vision. There were four subjects each in both Experiments 1 and 3; Experiment 2 used six subjects.

\section{Stimuli and Apparatus}

The targets were disk shaped and contained alternating

This research was supported by a grant from the National Eye Council, NIH, to L. Lefton (1 RO1 EY 01201-02). No official endorsement of NEI should be inferred. We thank Terry B. Or for help in data collection and analysis. Requests for reprints should be sent to the first author. square-wave black and white stripes. Varying spatial frequencies were used: $1.68,2.5,5,8,16$, or 19 cycles/deg of visual angle. The black stimuli were photoprinted on white semigloss paper to maximize contrast. The targets were $1.9 \mathrm{~cm}$ in diam and at a distance of $86 \mathrm{~cm}$ subtended $1.27 \mathrm{deg}$ of visual angle. The black ring used as a mask was $.87 \mathrm{deg}$ in width, inner to outer border. The annulus was $4.3 \mathrm{~cm}$ in diam at the outer edges and subtended $2.87 \mathrm{deg}$ of visual angle; there was no intercontour distance. Target and mask appeared directly over a centrally located fixation spot. The stimuli were presented monocularly to the right eye in a Gerbrand's four-field tachistoscope (Model T-4B).

\section{Procedure}

Upon introduction to the laboratory, subjects were dark adapted for $10 \mathrm{~min}$ and each subject helped the experimenter precisely align the target and mask. This procedure was repeated at the beginning of each experimental session.

Upon signal from the experimenter, subjects initiated their own trials by pressing a switch. The following sequence of stimulation was then presented: fixation, target, interstimulus interval, mask, and fixation. During the interstimulus interval the light fixation field was presented; there were never any dark intervals. Seven interstimulus intervals were used: $0,10,40,60$, 80,100 , and $500 \mathrm{msec}$.

\section{EXPERIMENT 1}

\section{Procedure and Design}

The subjects' task was to make a forced-choice decision as to the direction of orientation of the internal contours of the target. The contours were oriented such that the cycles ran $45 \mathrm{deg}$ to the left or the right of vertical. Two stimuli were prepared and placed in separate fields of the tachistoscope.

The subjects were tested at two target durations, 5 or $10 \mathrm{msec}$. Mask duration was constant at $200 \mathrm{msec}$. Subjects were instructed to respond "left" or "right" to the orientation of the target and to guess when necessary. The background luminance of the stimuli was equated to $50.2 \mathrm{~cd} / \mathrm{M}^{2}(14.7 \mathrm{fc})$.

During a single testing session, each subject saw targets of the same number of internal contours at one target duration. A session consisted of 12 blocks of trials. A block of trials consisted of a random ordering of the 14 experimental conditions: seven interstimulus intervals by two orientations. The first two blocks of each session were considered practice. Thus, each subject at the end of a session would provide 20 observations at each interstimulus interval ( 10 blocks by 2 trials per block); this was a totally within-subject design.

Following two full practice sessions, data were collected from each subject for 20 experimental sessions, with two sessions 
run for each of the five internal contour conditions at each duration. The highest contour target $(19 \mathrm{cycles} / \mathrm{deg})$ was not used, as pilot data showed it to be virtually indiscernible. The order of contours and durations was randomized and counterbalanced across subjects and sessions. This yielded a total of 40 measures per interstimulus interval per contour condition per target duration per subject.

\section{Results and Discussion}

The mean accuracy (percent correct above chance, defined as $50 \%$ correct discriminations) for each interstimulus interval for each contour condition for each target duration for each subject was determined and entered into analysis of variance. The analysis showed significant main effects of delay of mask $[F(6,18)=9.85, p<.001]$, internal contours $[\mathrm{F}(4,12)=75.57, \mathrm{p}<.001]$, and duration $[F(1,3)=146.60, p<.001]$. In addition, there were significant interactions of Delay of Mask by Internal Contours $[\mathrm{F}(24,72)=2.84, \mathrm{p}<.001]$, Delay of Mask Duration $[F(6,18)=8.67, p<.001]$, and Internal Contours by Duration $[\mathrm{F}(4,12)=5.82, \mathrm{p}<.01]$. The threeway interaction of Delay of Mask by Internal Contours by Duration was marginally significant $[F(24,72)=1.67$, $\mathrm{p}<.05]$. Data from Experiment 1 are shown in Figure 1 .

The extent of metacontrast was directly related to the number of internal contours. Targets with many internal contours were more difficult to mask than targets with few. Indeed, at the stimulus durations tested the 16-cycle stimulus was never discriminated well. The 500-msec delay of mask showed that the different contour targets are not seen equally well. A target with many contours is more difficult to discriminate even when it is not masked; thus, the relative amount of masking for these targets is limited. By contrast, targets with few internal contours were more easily masked, particularly at the 5-msec target duration; at the shorter interstimulus intervals the middle contours targets (5 and 2.54 cycles/deg) were less easily masked than was the lowest (1.68) contour target.

The target duration affected the extent of metacontrast markedly. Whenever metacontrast was obtained, monotonic functions were evident. However, there were dramatic floor effects in the 5-msec condition and similar ceiling effects in the $10-\mathrm{msec}$ condition.

The subjects were asked to perform a discrimination task. They were required to discriminate whether internal contours were oriented to the left or right. This is a relatively difficult task, particularly with a large number of contours. Again, this was shown by the relatively low accuracy for discrimination of the high contour targets when the mask was delayed 100 or $500 \mathrm{msec}$. Since the results showed marked ceiling and floor effects, Experiment 2 was designed to determine if these high or low contour targets are more or less difficult to mask. In Experiment 2, the same stimuli and apparatus were used; only the task was different-detection, with the dependent variable being duration threshold.

\section{EXPERIMENT 2}

\section{Procedure and Design}

The target stimulus was presented to the subject on each trial

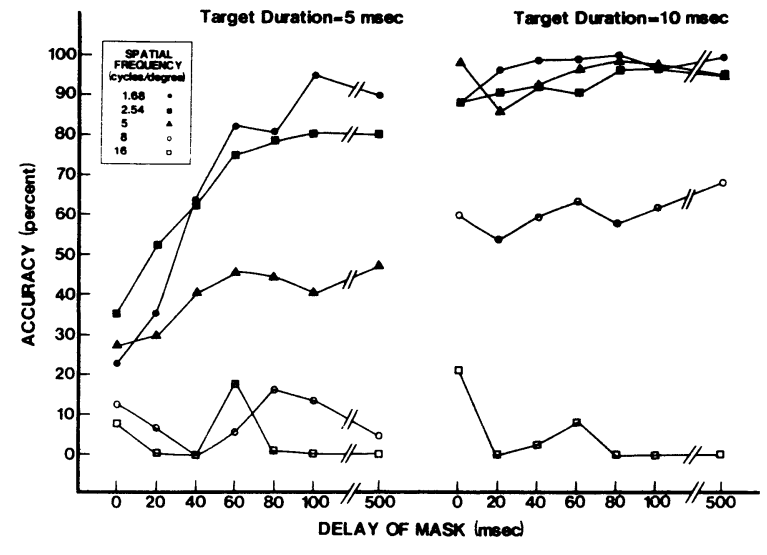

Figure 1. Results of Experiment 1. Accuracy is presented corrected for chance for all conditions. Each data point represents the mean of 160 observations.

for varying durations. The subjects were told on each trial to answer "yes" or "no" indicating whether or not they had seen the target. As the purpose was to determine the relative ease or difficulty of masking the various internal contour targets, rather than to establish absolute duration threshold values, the thresholds were obtained using the ascending method of limits with a criterion of three successive trials where the subject had answered that he had seen the target. Threshold was taken as the lowest duration of the three successive trials. A testing session consisted of 28 measurements, four ascending series for each of the seven interstimulus intervals. The 28 ascending series were randomized and counterbalanced within a session. Two practice series preceded each session, and prior to the start of the experiment each subject completed two full experimental sessions which were considered practice.

During a single session the subject saw targets of only one contour condition. Each of the six contour targets was tested for two sessions; order of targets was randomized and counterbalanced across subjects. Thus, each subject served for 12 experimental sessions, yielding eight duration thresholds per interstimulus interval per contour target per subject. Subjects were tested at two luminance levels, with half of the data at a high background luminance level $\left[50.2 \mathrm{~cd} / \mathrm{M}^{2}(14.7 \mathrm{fc})\right]$ and half at a lower luminance $\left[3.15 \mathrm{~cd} / \mathrm{M}^{2}(.92 \mathrm{fc})\right]$.

\section{Results and Discussion}

A mean duration threshold was calculated for each subject for each condition and entered into an analysis of variance. As in Experiment 1 there was a significant effect of internal contours $[F(5,30)=33.62, p<.011]$. As the mask was delayed, duration thresholds decreased $[F(6,36)=45.21, p<.001]$. The effect of luminance did not reach significance $[F(1,6)=2.17]$; however, the different internal contour targets were not detected equally well at the two luminance levels, as indicated by the interaction of Internal Contours by Luminance $[F(5,30)=5.08, p<.002]$. While the effect of the delay of mask was significant, this effect also interacted with luminance $[F(6,36)=11.12, p<.001]$, internal contours $[\mathrm{F}(30,180)=19.75, \mathrm{p}<.001]$, and with internal contours and luminance $[F(30,180)=14.04, p<.001]$.

When contrast in the targets was decreased, by decreasing luminance, targets with many contours were more easily masked than those with few contours. The mean duration thresholds for the high contour targets was significantly greater at 0 ISI than at 500 (Figure 2). 
A separate analysis for the $500-\mathrm{msec}$ delay showed that there were significant effects of contours such that higher contour targets were more difficult to see; this effect interacted with luminance, showing that at the lower luminance the effect was more pronounced. As in Experiment 1, higher contour targets are most difficult to detect even in a no-mask condition.

For bath luminance levels, the least amount of metacontrast at any interstimulus interval was obtained for targets of frequencies between 2.54 and 8 cycles/deg. With both fewer and more internal contours the extent of metacontrast was greater. The same effect was apparent, although not dramatic, in Experiment 1 at the 5 -msec target duration.

Overall, the results from this experiment show that, when using duration thresholds as the dependent variable, targets with both high and low numbers of internal contours are less easily detected. In addition, the interaction of Internal Contours by Delay of Mask shows that high and low contour targets are more susceptible to metacontrast. The triple interaction involving luminance shows that the effect becomes even more pronounced at lower luminance levels. The U-shaped functions of metacontrast over internal contours shows that the subject seems most sensitive to grating targets in the range of 2.5 to 8 cycles/deg and shows the least amount of metacontrast within this range.

To further explore the susceptibility of square-ware gratings to metacontrast, the same stimuli were used in Experiment 3 . The dependent variable was a category estimation of the extent of the clarity of a stimulus.

\section{EXPERIMENT 3}

\section{Procedure and Design}

The subjects were instructed to make a category estimation of the clearness of the presentation of the target. The estimation was based on a scale of 1 to 7 , with 1 being "absolutely clear." It was explained that a rating of 1 on any trial meant that the target was bold, clearly depictable, and the contours clearly defined. The target was presented for $13 \mathrm{msec}$ followed by a $200-\mathrm{msec}$ presentation of the mask. The luminance of the background of the field was equated at $12.5 \mathrm{~cd} / \mathrm{M}^{2}(3.4 \mathrm{fc})$.

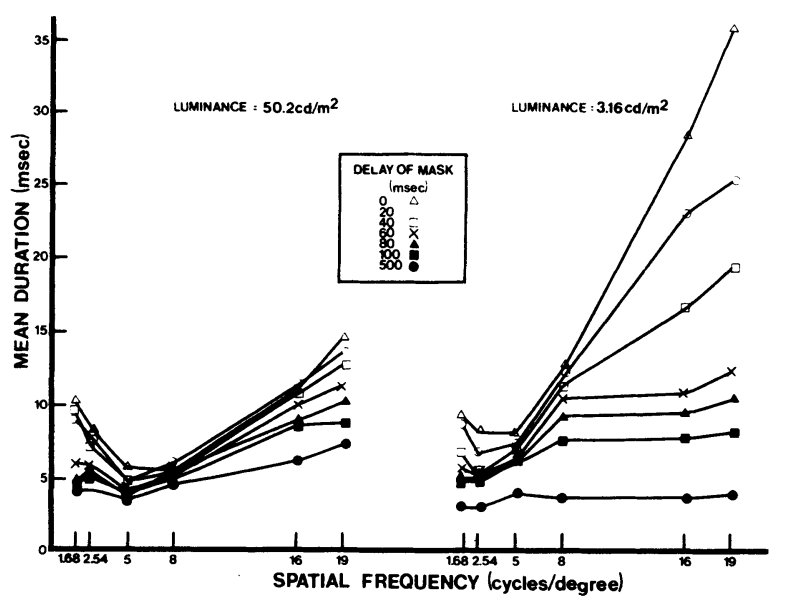

Figure 2. Results of Experiment 2. Mean duration threshold is presented for all conditions. Each data point represents the mean of 48 observations.
There were 15 experimental sessions per subject. The first three sessions were considered practice. The remaining 12 sessions consisted of 2 sessions devoted to each of the six contour targets. Within a session, each of the seven interstimulus intervals was presented 15 times for the subject to make category estimations. The order of the resulting 105 trials was randomized. Thus, at the end of the experiment each subject had contributed 30 measures at each interstimulus interval for each contour condition (15 measures per session $\times 2$ sessions).

\section{Results and Discussion}

A mean category estimation measure for each of the interstimulus intervals for each internal contour condition for each subject was computed and entered into an analysis of variance. With increases in the delay of the mask, the subjects reported a clearer stimulus $[F(6,18)=$ $35.65, \mathrm{p}<.001]$. The internal contours of the target also produced strong effects on detection such that high and low contour targets were less clear and, therefore, produced a larger overall category estimation $[F(5,15)=10.15, \mathrm{p}<.001]$. As in Experiments 1 and 2 there was a significant interaction of Delay of Mask by Internal Contours $[\mathrm{F}(30,90)=1.68, \mathrm{p}<.05]$.

Even when a mask was delayed $500 \mathrm{msec}$ so that it essentially had no effect, targets with higher numbers of internal contours were reported being less clear, a result consistent with those of Experiments 1 and 2 . In addition, when a mask was presented such that it produced metacontrast, the higher contour targets were more susceptible to metacontrast than the lower contour targets. As in Experiment 2 there was a U-shaped function of number of contours and the extent of detection. At grating frequencies between 2.54 and 5 cycles/deg there was markedly less metacontrast. Overall, using a markedly different dependent variable, Experiment 3 confirmed the results of Experiments 1 and 2 which showed that the extent of metacontrast is particularly dependent upon the internal contours of the target presented so that a U-shaped function is obtained.

The interaction of Internal Contours by Delay of Mask in Experiment 2 is particularly interesting. When category estimations were used as the dependent variable, the middle range frequencies produced monotonic functions as the mask was delayed. However, both the highest and lowest contour targets showed evidence for nonmonotonic U-shaped metacontrast functions. As is shown in the right-hand panel of Figure 3, subjects reported that these targets were more difficult to see at the $20-\mathrm{msec}$ delay of the mask compared to the $0-\mathrm{msec}$ delay. This means that subjects were reporting that the clarity of the target at these delays was lower than at 0 or at $60 \mathrm{msec}$.

\section{GENERAL DISCUSSION}

Several important findings are evident from these three studies. First, as targets have more or less internal contours, they are more susceptible to masking. This is particularly true with targets with many internal contours where limits of visual acuity cause high-frequency gratings to be far more difficult to see than ones of 5 to 8 cycles/deg of visual angle. Using accuracy, detection, and clarity measures, the same finding was obtained. 


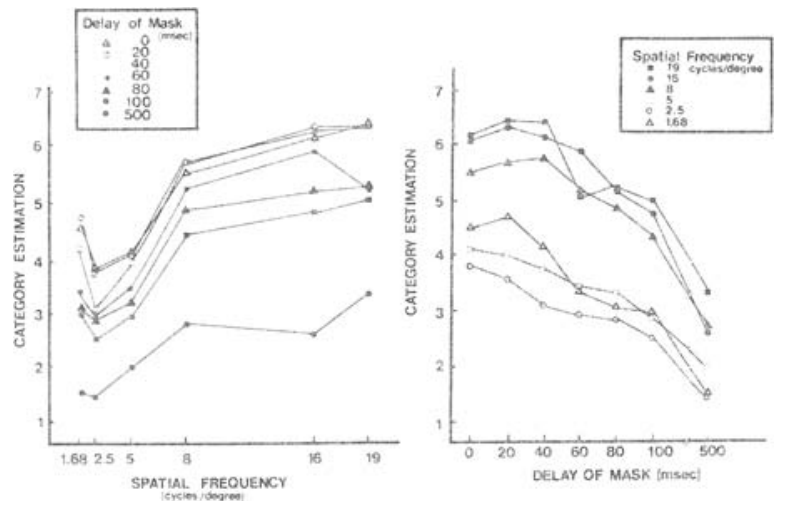

Figure 3. Results of Experiment 3. Mean category estimation is presented for all conditions. The lower the category estimation, the clearer the stimulus. The left panel shows the effects of spatial frequency most clearly. The right panel shows the evidence for monotonic and $U$-shaped functions. Each data point represents the mean of 120 observations.

This finding replicates some of the work of Dember and Lefton (e.g., Dember, et al., 1973; Lefton, 1974). However, in no condition was there any type of backward enhancement effect. Dember has reported that when a ring follows a target, particularly at high spatial frequencies, the target becomes easier to see than in its nonmasked state. The results of the present experiment show that when comparing a masked target to the $100-$ or $500-\mathrm{msec}$ delay condition, there are no enhancement effects. Quite the contrary, with many internal contours (and with very few) it is easier to produce metacontrast. Again, this has been shown using three different dependent measures at different interstimulus intervals.

Dember's experiments all used only the $0-\mathrm{msec}$ interstimulus interval. While no enhancement effects were obtained, the results of the present experiment support those of Dember by showing that targets with many internal contours are more difficult to detect. Further, they extend these findings to a lower range of internal contours and show that some of these are more susceptible to masking than those of the middle range. A comparison of some of the results of Dember (Dember et al., 1974) and those of the present study show markedly similar results over the range of grating targets tested.

One of the main questions of the present study was to compare the three different dependent variables in a metacontrast paradigm when the same stimuli and conditions were used. All three experiments showed similar results with regard to internal contours. High and low contour targets were more easily masked. These results parallel those reported when describing the basic sensitivity of the visual systems to spatial frequency gratings ${ }^{1}$ (Cornsweet, 1970).

With the three different tasks that were provided to subjects, there were some important differences. In both Experiments 1 and 2, where accuracy and duration thresholds were used, monotonic metacontrast functions were obtained. As the mask was delayed, accuracy increased or duration threshold decreased. This is a result that has been found repeatedly in a number of experiments (e.g., Eriksen, Becker, \& Hoffman, 1970; Lefton, 1973a, b, 1974). However, Experiment 3 produced some U-shaped metacontrast functions. The extent of this U-shaped function was not overly dramatic. Yet, these are clearly nonmonotonic. These are the first nonmonotonic masking functions that Lefton and his colleagues have been able to produce. The only major difference between Experiments 1 and 2 and Experiment 3 is the task that the subjects were asked to perform. When the subjects were asked to give a category estimation, the high and very low contour targets showed the U-shaped functions as the mask was delayed.

Both Lefton (1973b) and Schurman (1972) have argued that the critical variable between monotonic and U-shaped functions may be task differences. Schurman argued that U-shaped meta- contrast functions are not obtained with recognition or detection tasks. However, both U-shaped and monotonic functions have been obtained in both recognition and detection tasks. The difference between Experiments 1 and 2 and Experiment 3 in the present series of studies supports Schurman's contention.

One final note, Lefton and Griffin (1976) have recently reported a study of metacontrast with internal contours. They found that when target detectability was equated, using an accuracy measure, the effects of internal contours on metacontrast were totally eradicated. These were, of course, markedly different stimuli than the square-wave gratings used in the present study, and the experiment was limited by only using accuracy measures. The generality of those findings needs to be explored with grating targets using different types of dependent measures. If the extent of metacontrast with internal contour targets is diminished when target detectability is equated, then, as Lefton and Griffin have argued, targets with internal contours are less interesting. In any case, the difference between the dependent variable used, in and of itself, is sufficiently important to warrant further experimentation.

\section{REFERENCES}

Cornsweet, T. N. Visual perception. New York: Academic Press, 1970.

Cox, S. I., \& DEMBER, W. N. Backward masking of visual targets with internal contours. Psychonomic Science, 1970, 19, 255-256.

Dember, W. N., \& Stefl, M. Backward enhancement? Science, 1972, 175, 93-95.

Dember, W. N., Mathews, W. D., \& Stefl, M. Backward masking and enhancement of multisegmented visual targets. Bulletin of the Psychonomic Society, 1973, 1, 45-47.

Dember, W. N., Stefl, M., \& KaO, K. C. Backward masking of gratings varying in spatial frequency. Bulletin of the Psychonomic Society, 1974, 3, 439-441.

Ellis, D., \& DEMBER, W. N. Backward masking of visual targets with internal contours: A replication. Psychonomic Science, 1971, 22, 91-92.

Eriksen, C. W., Becker, B. B., \& Hoffman, J. E. Safari to masking land: A hunt for the elusive U. Perception \& Psychophysics, 1970, 8, 245-250.

KeLly, D. H. How many bars make a grating? Vision Research, 1975, 15, 625-626.

Lefton, L. A. Spatial factors in metacontrast. Perception \& Psychophysics, 1973, 14, 497-500. (a)

Lefton, L. A. Metacontrast: A review. Perception \& Psychophysics, 1973, 13, 161-171. (b)

LEFTON, L. A. Internal contours, intercontour distance, and interstimulus intervals: The complex interaction in metacontrast. Journal of Experimental Psychology, 1974, 103, 891-895.

Lefton, L. A., \& Griffin, J. Metacontrast with internal contours: More evidence for monotonic functions. Bulletin of the Psychonomic Society, 1976, 7, 29-32.

Schurman, D. L. Predictive validity of a Rashevsky-Landahl neural net: Test of a model of masking for form. Perception \& Psychophysics, 1972, 12, 183-186.

VAN DEN BRINK, G., \& BILSEN, F. A. The number of bars that makes a grating for the visual system: A reply to Dr. Kelly. Vision Research, 1975, 15, 627-628.

\section{NOTE}

1. There has recently been a controversy over the number of bars that make a grating. Kelly (1975) and van den Brink and Bilsen (1975) have argued the issue of the minimum number of bars necessary to provide an adequate spatial frequency grating. Gratings which have a limited number of bars, such as those used in the present experiment, may limit the generality of these findings. In the present experiment this would be particularly true with the low spatial frequencies. The issue is not yet resolved. 